\title{
ENTRE ROSALÍA 21 Y LABREGOS DO TEMPO DOS SPUTNIKS: LA POESÍA GALLEGA COMO ESPECTÁCULO ${ }^{1}$
}

\section{Isaac LOURIDO HERMIDA}

Grupo Alea de Análise Poética - Universidade de Santiago de Compostela

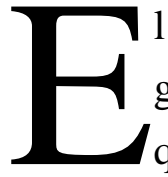

desarrollo de un espacio cultural autónomo y legitimado verifica en el caso gallego algunos de los condicionantes que se han manejado para escenarios en que concurren sistemas culturales de distinto vigor, consolidación y reconocimiento histórico. Así, frente a una cultura oficial e indiscutida, difundida desde las instituciones encargadas de consolidar un modelo de lo español nunca suficientemente plural ni atento al valor de la diferencia, los/as agentes partidarios/as de la emergencia e institucionalización de una cultura gallega útil y reconocible para su ciudadanía se han pasado las cuatro últimas décadas respondiendo preguntas ${ }^{2}$.

Atienden éstas básicamente a las condiciones para la superación de un estado de dependencia (institucional y simbólica) que siempre ha contado con correlatos políticos de diverso signo; a la tensión entre lo tradicional y lo innovador, con matices que van desde la tentación folclorizante a la apuesta por maniobras de construcción e invención

\footnotetext{
${ }^{1}$ Este trabajo está vinculado con el proyecto de investigación El discurso no lírico en la poesía contemporánea: Espacios, sujetos, hibridación enunciativa, medialidad, que cuenta con financiación pública del Ministerio de Ciencia e Innovación del Gobierno de España (FFI2009-12746). Una versión previa fue desarrollada en el blog http://poesiagalegoaikuskizun.wordpress.com. Este blog funciona también como repositorio de materiales textuales, audiovisuales e hipermedia relacionados con los asuntos que aquí son estudiados.

${ }^{2}$ En todo el texto se toma como referencia marco el conjunto de la denominadas teorías sistémicas. Es decir, aquellas propuestas que, como la teoría de los polisistemas, desarrollada por Itamar Even-Zohar (1990, 2005), o la teoría del campo literario, debida a Pierre Bourdieu (1991, 1992), consideran que el estudio de lo literario debe superar modelos de análisis centrados en lo textual o lo estilístico para considerar la literatura como un conjunto de prácticas y relaciones, organizadas de acuerdo con parámetros específicos funcionales y de jerarquización, y sometidas a dinámicas constantes de cambio provocadas por las luchas destinadas a la consecución de los capitales (económicos, simbólicos o culturales) que se ponen en juego en torno a la actividad literaria. Algunas aplicaciones preliminares de las teorías sistémicas al campo literario gallego desde 1975 fueron efectuadas por Xoán González-Millán (1994) y Antón Figueroa (2001).
} 
desacomplejadas; a la relación con una Historia cuyo relato se ha considerado mayoritariamente problemático e indigno al mismo tiempo que heroico y traumático, pero también por eso abierto a la reescritura; o, en definitiva, a la complejidad de asumir con suficientes evidencias todas las relaciones establecidas entre dispositivos culturales, políticos e identitarios, circunstancia que en el peor de los casos lleva a la preeminencia de lo cultural, a la postergación indefinida de lo político y al estatismo más absoluto en relación con cualquier renegociación de lo identitario.

En este cuadro de análisis deben ser entendidas las funciones desarrolladas por la literatura gallega históricamente, y de manera particular en los procesos que acompañaron el final de la dictadura franquista y la reforma política que desembocó en el actual marco jurídico-institucional del Estado español. Algunos de los factores que no pueden ser obviados para una comprensión más precisa de la convergencia entre poesía, espectáculo y cultura gallega en la contemporaneidad se relacionan con la incorporación de la lengua gallega como elemento simbólico privilegiado en la reconfiguración identitaria, la consagración de la literatura como discurso artístico central y conductor de los debates políticos que no siempre contaron/cuentan con el foro adecuado para su deliberación y, finalmente, la preeminencia de la poesía en relación con los otros géneros literarios. La articulación de este último factor se corresponde con la referencia a determinados monumentos historiográficos (como la lírica medieval galegoportuguesa y el Rexurdimento poético de la segunda mitad del siglo XIX) y con la adjudicación de determinadas atribuciones y funciones al género desde el Romanticismo, vigentes aún en aquellos procesos inacabados y en curso.

En este trabajo se aspira a proponer determinados horizontes para la identificación y el estudio de las tensiones y de los principios de visión y división que estructuran el campo poético gallego en la actualidad. Desde este punto de vista, se antoja necesaria una referencia constante a los distintos procesos de institucionalización y, más concretamente, a la contraposición en este terreno de las estrategias propuestas en torno a dos polos fundamentales: el de la normalización y el de la diferencia -cultural, identitaria y política- (González-Millán, 2000). Asimismo, el análisis de los modos en que las culturas denominadas periféricas, subalternas y/o emergentes reciben y asimilan los flujos emanados desde distintos centros de producción cultural, cuyo alcance adquiere relevancia internacional, parece de referencia obligada para afinar $o$ contradecir marcos teóricos y analíticos propuestos como universales.

En un enfoque que pretende combinar la observación del conjunto con la atención a fenómenos y experiencias concretas, han sido marcados tres ámbitos de atención básicos: la presencia de la poesía oral en el espacio público gallego, abordada en perspectiva histórica; las relaciones establecidas entre lo poético y lo musical con 
especial atención para los modelos y funciones activados; y la atención específica a tres proyectos poético-musicales contemporáneos (la iniciativa interdisciplinar Rosalía 21, la sincrética propuesta musical de Labregos do tempo dos sputniks y la trayectoria del grupo de pop electrónico Fanny + Alexander) que, en sus especificidades y diferencias, permiten incrementar el número de claves para la comprensión de un mapa inconcluso, móvil, tal vez en construcción.

\section{Poesía, oralidad y espacio público}

La fórmula del recital ha venido siendo la más socorrida para introducir la poesía manifestada oralmente en el espacio público gallego, por lo menos en las cuatro últimas décadas. Se trata de una fórmula ambivalente y que, en principio, no parece prudente ligar a una funcionalidad específica ni a proyectos de planificación o intervención cultural concretos. Por un lado, ha gozado de cierta rentabilidad en sus vínculos con determinada figura del escritor/poeta (casi nunca la escritora/poeta) definida por su compromiso político y con la confianza depositada en este tipo de intervenciones como práctica de movilización social. Pero también ha sido frecuente su presencia en actos pensados a partir de concepciones estáticas (esencialistas o folclorizantes) de lo lírico o lo cultural, no siempre, por cierto, restringidas al espacio privado o a auditorios restringidos. Entre un extremo y otro, lógicamente, ha habido oportunidad para la apertura de nuevas expectativas y modos de participación, no sólo para los/as poetas sino también para el público.

Se trata de un modelo, el del recital, ampliamente representado en la década de 1970 y en la primera mitad de 1980, justamente cuando el emergente campo cultural gallego, en relación dialéctica con los acontecimientos que transformaban el panorama político e institucional en España y en Galicia, acumulaba en su interior grandes dosis de energía y dinamismo. Sólo a partir de un cierto estancamiento de esta dinámica emergente, vinculado a lo que se ha venido a denominar el proceso de normalización cultural, es necesario hablar de una progresiva institucionalización (e incluso ritualización) de este tipo de actos. Por ejemplo en aquellos organizados por la Asociación de Escritores en Lingua Galega o por la sección gallega del Pen Club en la última década, con una disposición más definidamente sectorial y con una vocación pública y política neutralizada, tal vez inane, en muchas ocasiones.

Estos cambios y reorientaciones no pueden ser desvinculados de debates que vienen de antiguo. Por ejemplo, en torno a la confianza o desconfianza en la posibilidad de que la poesía asuma responsabilidades políticas, el descrédito generalizado de los repertorios propuestos por la poesía social, la trascendencia atribuida a la labor de la 
Entre Rosalía 21 y Labregos do tempo dos sputniks...

escritura (en una escala que va de la sacralización a la parodia) o la identificación de lo normal con la separación entre arte y política. En este sentido, la aludida reconfiguración de la tipología y la funcionalidad de los recitales se desarrolló en tensión con opciones que pujaban por la actualización del discurso poético en su representación pública, mediante la introducción de claves subversivas e iconoclastas en términos políticos y estéticos, por ejemplo a través de prácticas performativas y musicales que casi siempre contaron con el humor como ingrediente necesario. Frente al rodillo normalizador, todas estas claves están presentes en las trayectorias de los colectivos Rompente y Ronseltz, activo el primero ya a finales de los 70 y el segundo a mediados de la década siguiente ${ }^{3}$.

Otras rupturas con el modelo más convencional del recital, el del vate posicionado frente a su auditorio en espacio "adecuado", se produjeron durante la década de 1990, de acuerdo con la introducción de repertorios en que lo político-identitario recobraba protagonismo y con la incorporación definitiva de las mujeres al campo de la creación poética ${ }^{4}$. Las renovaciones fundamentales operadas en esta década se relacionan con la traslación de los recitales a espacios menos frecuentados y no siempre previstos, básicamente determinados bares y pubs de Compostela, de acuerdo también con los ritmos de algunas publicaciones emergentes en la época articuladas al compás de la masiva integración de la juventud gallega en la Universidad. Como siempre, como en todo relato más o menos histórico, había aún algunas otras cosas: no muy desconectado de este ambiente, fue relevante la actividad editorial y activista del Batallón Literario da

\footnotetext{
${ }^{3}$ Rompente, fundado en 1975 en Vigo por Alfonso Pexegueiro y Antón Reixa, tuvo como nómina más estable de integrantes al propio Reixa, a Manuel M. Romón y a Alberto Avendaño. Resultado de la dialéctica entre renovación estética y agitación política, Rompente combinó las iniciativas de carácter propiamente textual, algunas firmadas colectivamente como Silabario da turbina (1978), con la apertura progresiva al discurso performativo, siempre con la parodia y la trasgresión de los nuevos límites de lo poético que se intentaban instaurar como objetivos últimos. Su último espectáculo fue A tristeza de Ezza (1982). El colectivo Ronseltz, por su parte, fue creado en A Coruña en 1985 y estuvo integrado por Xoán C. Rodríguez, Sérxio Iglesias, Miguel A. Montes, Xabier Cordal y Manuel Cortés. Insistió en buena parte de las estrategias exhibidas por Rompente, tal vez con un registro menos variado y audaz. En 1994, con el grupo ya inactivo, se publicó una antología de sus textos (Unicornio de cenorias que cabalgas os sábados, Compostela, Edicións Positivas).

${ }^{4}$ Es un lugar común en la historia literaria y la crítica la representación de la producción poética gallega de las décadas de los 80 y los 90 como la de dos movimientos literarios antitéticos. El primero de ellos fue caracterizado como partidario de la renovación de los repertorios poéticos de acuerdo con valores opuestos a los de la poesía social y de la apuesta por determinados objetivos literarios sublimados en torno a categorías del gusto occidental hegemónico como la belleza, la excelencia o la trascendencia estética. La poesía de los 90, por el contrario, se describe habitualmente como aquella que recuperó cierto pulso cívico y comunitario y que, por otro lado, mostró su permeabilidad a determinadas reconfiguraciones teóricas y discursivas, como el feminismo o la cultura de masas. Visiones menos maniqueas insisten en destacar la presencia de repertorios compartidos entre las producciones de ambas décadas, así como la necesidad de ajustar los análisis de acuerdo al funcionamiento de determinados grupos y colecciones editoriales, las dificultades para articular un reconocimiento preciso de lo central y lo periférico, la importancia de determinadas localizaciones geográficas o, entre otros, los procesos dialécticos establecidos entre escritura poética y las distintas propuestas de planificación cultural y política vigentes en cada período (Cochón, 2001).
} 
Costa da Morte, empeñado en descompensar la centralidad urbanita de lo poético y en crear nuevas fórmulas de engarzar lo poético con lo popular, con frecuencia en actividades co-presenciales y multiparticipativas.

Algo de todos estos modelos que han sido expuestos persiste en la actualidad. Persiste, desde luego, la fórmula más convencional del recital concebido como mera lectura de poemas en determinados actos culturales, sociales y políticos. No es infrecuente, por otro lado, la organización de recitales en bares o pubs, aunque quizás con menos dinamismo que en la década de los 90 e, incluso, en algunas ocasiones con planificación a cargo de las instituciones políticas de turno. Por otro lado, el terremoto político y cultural organizado en torno al movimiento Nunca Máis (que contestó la gestión política del hundimiento del petrolero Prestige frente a las costas gallegas en noviembre de 2002) pareció hacer recuperar la confianza en la capacidad no sólo de la poesía, sino del arte en general, para provocar nuevas cadencias en el ámbito político. Entre otras repercusiones, permitió la recuperación de cierto espacio para la poesía en la calle, articulada por ejemplo en actos como los convocados por los colectivos Mares de Tinta y Redes Escarlata, y con continuidad hoy en día en eventos como la Semana da Poesía Salvaxe, en Ferrol. Se trata de una convergencia, la de poesía y política, con la que lleva años haciendo un camino histórico el Festival da Poesia no Condado, celebrado en esa comarca del sur de Galicia desde 1981 y referente indiscutible para muchas de las cuestiones que aquí se abordan.

Otras aperturas de las que es necesario dejar constancia tienen que ver con la integración multimedia de lo poético, desarrollada por Antón Reixa ya en la década de los 90 (por ejemplo en el disco Escarnio o en el espectáculo Galicia Beibe, ambos de 1999); la dramatización o parateatralización del poema como fórmula en que lo poético y lo teatral apuntalan su discurso en el escenario público, con nombres como los de Antón Lopo (Prestidixitador, poemario oral, 2001), Ana Romaní (Lob*s, 1998, con el propio Lopo) o Lupe Gómez (Porno, 2002); o la aproximación entre discursos poéticos contemporáneos como el slam poetry o el hip-hop y propuestas de base tradicional, sean éstas de carácter musical o propiamente orales, como la regueifa. Algunas convocatorias recientes en esta línea son el Certamen Internacional de Improvisación Oral e Regueifas (Vigo, octubre de 2009) o el Festival Xalleiro de Regueifas e Hip-hop (Santa Comba, octubre de 2009). También han tenido cierto protagonismo lo oral y lo poético en varias iniciativas de la "comunidad de arte y acción libre" Alg-a (Sopa de Alg-as, Valadares - Vigo, febrero de 2010) ${ }^{5}$.

\footnotetext{
5 Información sobre este y otros eventos puede ser consultada en la página web del colectivo: www.alg-a.org [última consulta: 14-9-2011].
} 
Entre Rosalía 21 y Labregos do tempo dos sputniks...

\section{Poesía y música}

Buena parte de la sucesivas revisiones de la fórmula recital quisieron contar con el factor musical como elemento que permitiese hacer más atractivas unas propuestas de base claramente literaria. Y aunque la introducción de la música no supuso en muchos casos más que un mero "acompañamiento" sin verdaderos efectos transformadores a nivel discursivo y performativo, merecen ser destacados algunos proyectos $\mathrm{y}$ espectáculos en los que se aspira a la comunicación de algo más que un poema que, en la encrucijada formada por lo poético-textual, lo musical y, eventualmente, lo político, resulte revelador y significativo en el campo cultural gallego.

Para entender en todo su sentido la rentabilidad de las propuestas que serán analizadas con mayor detalle, es necesario identificar y explicar brevemente los modelos que fueron más habituales para la incorporación de los textos poéticos al panorama musical gallego en las últimas décadas. El modelo más clásico sería el del cantautor (de nuevo, casi nunca cantautora), surgido en Galicia a finales de 1960 a rebufo de otros movimientos de canción protesta en la península y encajado en una industria musical muy precaria. El colectivo más significativo fue Voces Ceibes, vinculado con determinadas estrategias del Partido Comunista de España y cuyas composiciones contaban con los poemas sociales de la época, escritos en gallego, como letras más representativas. Esa incorporación de los textos poéticos a la canción de autor fue prolongada con posterioridad por otros músicos como Suso Vaamonde (ya presente en Voces Ceibes), Emilio Cao, Luis Emilio Batallán o Amancio Prada, cada uno con sus especificidades y desde parámetros sustancialmente distintos ${ }^{6}$.

Un segundo modelo reconocible fue el de la música folclórica, rechazada desde la izquierda en un primer momento por la apropiación degradante que de ella habían hecho las instituciones oficiales del franquismo. La influencia de referentes como el portugués, personificado en la figura de José Afonso, abrió progresivamente la puerta a la incorporación de los repertorios de la música tradicional y popular a una funcionalidad política y social, por ejemplo en relación con esa inscripción de la identidad gallega en

\footnotetext{
${ }^{6}$ Suso Vaamonde constituye el modelo prototípico del cantautor comprometido políticamente. Su voz y su guitarra sirvieron de altavoz para melodías austeras y letras siempre ajenas o de procedencia popular. Situado ideológicamente en la izquierda nacionalista, fue encarcelado a comienzos de los 80 por injurias a "los símbolos de la patria". Emilio Cao, por su parte, procuró la convergencia de la música tradicional gallega y otros ritmos procedentes del ámbito celta en una trayectoria que dura hasta nuestros días y durante la que no faltó la complicidad con la obra de poetas como Manuel Antonio o Uxío Novoneyra. Luis Emilio Batallán exhibió en el exitoso Aí vén o maio (1975), sobre textos de Curros Enríquez, Rosalía de Castro, Ramón Cabanillas, Celso Emilio Ferreiro y Álvaro Cunqueiro, una música arrimada al pop melódico, casi sin continuidad posterior. Finalmente, Amancio Prada, originario del Bierzo (territorio oficialmente leonés donde se habla gallego), ha combinado en su trayectoria la musicalización de la poesía de varios poetas gallegos (como Rosalía de Castro y Álvaro Cunqueiro) con la producción de otros discos nítidamente orientados a su distribución en el ámbito estatal, en una carrera en el que el perfil de cantautor nunca se quiso desvincular de la música folclórica.
} 
el ámbito del mundo celta inaugurada por Emilio $\mathrm{Cao}^{7}$. Un caso significativo de esa tensión entre el cariz pintoresco que el franquismo había adjudicado a la música folclórica y un compromiso de trazo emancipador, orientado claramente hacia este segundo polo, es el grupo Fuxan os Ventos. La conexión con lo poético se efectuó a menudo a través de letras escritas por autores como Manuel María o Celso Emilio Ferreiro, pero también con el aprovechamiento de poemas preexistentes y con la incorporación de composiciones procedentes de la tradición oral. Las tres posibilidades fueron seguidas por grupos como A Quenlla y Na Lúa. La segunda, aunque sólo de forma circunstancial, por Luar na Lubre o por el gaiteiro Carlos Núñez.

Tanto la canción de autor como la música folk identificaban como referente al que se oponían las propuestas musicales que, situadas en la órbita de la denominada canción ligera, mantenían unas aspiraciones de carácter básicamente comercial pese al uso más o menos constante de la lengua gallega. En la mayoría de los casos, como los de Juan Pardo o Ana Kiro (con grandísima aceptación en los centros gallegos radicados en los principales destinos americanos y europeos de la emigración gallega), el uso de la lengua o de determinados tópicos temáticos se usaban simplemente a partir de su carácter pintoresco y exotizante. Ejemplos más complejos son los de Andrés do Barro, homenajeado en 2007 en su faceta (pionera) de cantante pop en gallego, o el grupoorquesta Los Tamara, con el crooner Pucho Boedo a la cabeza, que incluían textos poéticos entre sus letras.

Al igual que en el caso de la poesía, la tensión con modelos distintos y alternativos a los dominantes provocó, ya desde finales de la década de 1980, la diversificación de las propuestas musicales, en muchas de ellas con una relajación de las funciones pragmáticas y políticas que se le suponían a los modelos de la canción protesta y de la música folk. Y en otros casos, como el del Movimiento Bravú, organizados en torno a una cierta base ideológica y generacional orientada a la reivindicación del mundo rural y de las periferias rural-urbanas en términos dinamizadores y de diálogo con los repertorios clásicos del rock y el punk. La colaboración con algunos escritores como Santiago Jaureguízar o Manuel Rivas no implicó, sin embargo, un uso frecuente de los textos literarios en las letras de las canciones.

Conviene no olvidar, en todo caso, los límites del campo en el que se ha venido jugando en todos estos años y que continúa plenamente vigente en la actualidad, por ejemplo en lo que tiene que ver con los modelos musicales mayoritarios (música comercial difundida a escala global desde las delegaciones españolas), con la solo

\footnotetext{
${ }^{7}$ La conexión entre la canción protesta gallega y la obra de José Afonso, así como su relevancia en la progresiva introducción de un interés progresivo por el folclore y la música popular, fue explicada con mayor profundidad por Maria Felisa Rodríguez Prado (2004).
} 
Entre Rosalía 21 y Labregos do tempo dos sputniks...

emergente pero aún no pujante consolidación de una escena musical gallega (y en gallego) y un público correlativo, o con dinámicas de apoyo-dependencia derivadas de lo político y lo institucional. Son factores que podremos ir analizando a partir de las tres propuestas que serán estudiadas de forma más específica, encaminadas a la convergencia de lo poético (en sentido textual) y lo musical en proyectos que aspiran a abrir caminos de renovación o alternatividad.

\section{Rosalía 21}

Rosalía 21 es un proyecto multidisciplinar, coordinado por el profesor y poeta Anxo Angueira, que se concreta en tres iniciativas de distinto perfil: un libro de estudios sobre Rosalía de Castro (Angueira, 2009), un disco y un espectáculo poético-musical ${ }^{8}$. Frente a las lecturas interesadas en resaltar la vertiente intimista de la poeta y frente a los tópicos que la asocian con una melancolía y una tristeza inútiles en sentido político, Rosalía 21 pretende la reactivación de análisis contemporáneos afines a las reivindicaciones nacional, feminista y de clase. Indagaciones todas que, desde cierto esquema intelectual, refuerzan no solo su centralidad en el canon sino también su rol como fundadora de la literatura gallega en la segunda mitad del siglo XIX y, por extensión, como fundadora de la nación gallega moderna.

Para explicar las dimensiones y el recorrido del proyecto es imprescindible dejar constancia del apoyo que tuvo por parte de la Consellería de Cultura del gobierno bipartito PSdeG-PSOE - BNG (2005-2009). Un apoyo que ayuda a explicar, por ejemplo, la ambición de la iniciativa, la cuidada producción del disco y, significativamente, que el estreno del espectáculo tuviese lugar en febrero del 2008 en La Habana, dentro del conjunto de actividades programado por la Consellería en la Feria del Libro de dicha ciudad, que contaba con Galicia como país invitado en esa edición ${ }^{9}$.

La dimensión poético-musical de Rosalía 21 puede ser analizada a partir de las trayectorias (también en el sentido bourdiano del término) que en él se concitan: las del compositor y pianista de jazz Abe Rábade, la cantante Guadi Galego y el propio Anxo Angueira como poeta y recitador. El primero de ellos, como figura formada $\mathrm{y}$ reconocida en el ámbito internacional, representa de alguna manera la más singular, por

\footnotetext{
${ }^{8}$ El disco Rosalía 21 fue editado bajo el sello de Falcatruada (2007) y contó con el apoyo económico de la Xunta de Galicia.

${ }^{9}$ Problemas técnicos frustraron su estreno en territorio gallego el 26 de febrero de 2009 en Santiago de Compostela. Tras la llegada del PP al gobierno (marzo de 2009), el espectáculo se ha representado solo de forma ocasional y casi siempre en un formato reducido. El estreno en Galicia tuvo lugar el 24 de febrero de 2010 en Santiago de Compostela, ya con otro nombre (Homenaxe a Rosalía) y sin mención en los anuncios del evento al proyecto original.
} 
escasamente frecuentada, de las elecciones que planean sobre el disco: la del jazz como discurso musical contemporáneo y emblema de la modernidad y del gusto selecto en las sociedades de consumo europeas. El diálogo con la tradición musical gallega ha constituido uno de sus centros de interés en los últimos años, por ejemplo en el proyecto Nordestin@s (con la cantante Ugia Pedreira y con la propia Guadi Galego).

La voz de Guadi Galego, por otro lado, es una de las más reconocidas en el ámbito de la música folclórica gallega. Cantante durante años del grupo Berrogüetto, continuó su trayectoria posterior en iniciativas como Espido (junto a la guitarra de Guillerme Fernández) o la ya mencionada de Nordestin@s. El perfil de Anxo Angueira, finalmente, es multifacético en sus dimensiones de poeta, profesor e investigador. Situado políticamente en la izquierda independentista afín a la red que constituyen la Frente Popular Galega, la Central Unitaria de Traballadores y el frente cultural Redes Escarlata (deudores todos ellos del magisterio de Xosé Luís Méndez Ferrín), el conjunto de su actividad profesional y pública remite a un programa político de corte emancipador. Así sus estudios en torno a figuras centrales del canon como la propia Rosalía, Eduardo Pondal o Méndez Ferrín, los repertorios exhibidos en una ya amplia obra literaria o, y esto es lo que más nos interesa, su creciente protagonismo como recitador/declamador de textos poéticos, casi siempre afinados en clave nacionalpopular y épica.

En el espectáculo, la música de Abe Rábade, compuesta específicamente a partir de nueve poemas de Rosalía de Castro, es interpretada por el cuarteto que lleva su nombre y se acompaña del canto de Guadi Galego y del recitado melódico de Angueira, casi siempre por separado ${ }^{10}$. En la selección de poemas se combinan algunos de los poemas más canonizados de la autora (como "Negra sombra") con otros que propician lecturas orientadas hacia los discursos emancipadores y combativos que antes fueron mencionados (así, “A xusticia pola man” o “Tecín soia a miña tea”)" posición esta última reforzada por la lectura que Angueira realiza de un fragmento del prólogo de Cantares gallegos (1863) como apertura del espectáculo ${ }^{12}$. La proyección de intervenciones de determinados/as escritores/as e investigadores/as sobre la obra de Rosalía en momentos concretos del espectáculo fue incluido en el estreno de La Habana, apuntalando algunas de las claves socio-sistémicas que sostienen el proyecto:

\footnotetext{
${ }^{10}$ Completan el Abe Rábade Quartet Jesús Santandreu (saxo tenor), Nelson Cascais (contrabajo) y Bruno Pedroso (batería).

${ }^{11}$ Los diez poemas incluidos en el disco e interpretados en el espectáculo son los siguientes: "A xusticia pola man", "Padrón, Padrón, Santa María, Lestrove", "Ladraban contra min", "Cada noite eu chorando pensaba", "Negra Sombra", "Tecín soia a miña tea", "Tembra un neno" (de Follas Novas, 1880); "Miña Santiña","Alborada” (de Cantares Gallegos, 1863), y “A Rosalía / Altares do Silencio”, de Luís Pimentel. 12 Iria Sobrino (2009) ha analizado la función manifestataria desarrollada por Rosalía de Castro en los prólogos de Cantares Gallegos y Follas Novas.
} 
Entre Rosalía 21 y Labregos do tempo dos sputniks...

la dependencia del campo político, la voluntad de inserción en una industria cultural normalizada y la actualización del canon poético nacional de acuerdo con una síntesis de repertorios intelectuales y culturales en que conviven lo moderno y lo posmoderno.

\section{Labregos do tempo dos sputniks}

El proyecto musical Labregos do tempo dos sputniks, integrado por O Leo i Arremecághona (guitarras y voz), García (voz y bases electrónicas) y Silverio Outeiro (batería y coros), puede ser definido a partir de dos claves principales: la reinscripción contemporánea del discurso literario de Celso Emilio Ferreiro y la conexión con los movimientos sociales más dinámicos y menos institucionalizados, ajenos a la lógica parlamentaria y a la dependencia institucional ${ }^{13}$. En cuanto a la primera de estas claves, la iniciativa parece tener entre sus intenciones no solo la reivindicación del poeta social gallego por excelencia de la época franquista sino también la ampliación y resignificación de un discurso que, en contra del tópico fosilizado por la crítica, nunca se restringió al prosaísmo más evidente y contó con una variedad de modos y registros en los que el humor, la experimentación y el diálogo intercultural tuvieron un protagonismo no menor ${ }^{14}$.

En relación con la inclusión de la iniciativa en el tejido más reivindicativo de los movimientos sociales de izquierda, es suficiente con observar algunas de las convocatorias en las que han colaborado a lo largo de una trayectoria que comenzó en 2007: Concerto pola Liberdade de la plataforma Que voltem à casa (de apoyo a los presos independentistas), Fiesta de despedida del centro social Casa Encantada, Fiesta del Cineclube de Compostela (con lema "La propiedad intelectual es un robo"), concierto en apoyo a la plataforma ecologista Galiza Non se Vende, Jornadas Zapatistas del Centro Social A Fouce y un larguísimo etcétera.

Como en el caso de Rosalía 21, una definición aún más exacta de su posición, que nos proporciona también el heterogéneo perfil musical del proyecto, puede ser trazada si entrecruzamos las trayectorias de los tres integrantes del grupo. El cantautor punk O Leo i Arremecághona ha venido desarrollando desde la última década un recorrido musical polimórfico en el que, con base en versiones de determinados clásicos del punk, se propone como motivo central la relectura en clave cultural y política de la identidad

\footnotetext{
${ }^{13}$ Utilizamos el concepto de reinscripción en un sentido no lejano del propuesto por Edward Said (1993) para la lectura de determinadas obras producidas en contextos poscoloniales. Es decir, como estrategia de contra-escritura consistente en la reapropiación subversiva de las representaciones y los discursos hegemónicos (de base específicamente colonial, en el argumentario de Said), orientada en última instancia a la construcción de una nueva autorrepresentación en clave de resistencia cultural.

${ }^{14} \mathrm{El}$ nombre del proyecto se corresponde con el último verso del irónico poema de Celso Emilio Ferreiro "Eu son un rei" (Longa noite de pedra, 1962).
} 
gallega. Con modelos como Albert Plà, Leo despliega en sus diversos espectáculos performativo-musicales la desintegración simbólica de imaginarios como el promovido por el nacionalismo español, blanco frecuente de sus parodias. Desde una posición, además, que tampoco es ajena al diálogo con cierta tradición musical propia (por ejemplo, con la trayectoria de Suso Vaamonde y, en general, con la canción de autor) que, en sus vínculos con lo literario, se ha preocupado de reivindicar, actualizar y versionar con infinitud de actuaciones por toda Galicia, principalmente en los ámbitos de la izquierda nacionalista.

Por su parte, el rapero García, miembro del grupo de hip-hop con mayor proyección en el ámbito gallego, Dios Ke Te Crew, compagina su dedicación a esta banda con el activismo político en determinados movimientos sociales inclinados hacia posiciones libertarias y articulados en torno a la denuncia del sistema carcelario o las luchas de las personas migrantes, sin olvidar sus vínculos con la plataforma ecologista Galiza Non Se Vende. De su querencia hacia el movimiento zapatista parece partir una sensibilidad específica hacia los repertorios (musicales y políticos) resistentes de América Latina, transferidos también al espectáculo de Labregos do tempo dos sputniks, y patentes en algunas de sus intervenciones en lo que podríamos denominar "acción directa". La última pieza del terceto es Silverio Outeiro, Vero, batería del histórico grupo Xenreira (Érguete, 1999) que, en su breve trayectoria, desde el ska se acercaba en algunos temas al hardcore y en otros al reggae y surgió claramente influenciado por determinadas bandas del ámbito vasco como Negu Gorriak. Con un perfil de militancia nacionalista más clásico, la incorporación de Vero a Labregos do tempo dos sputniks tuvo lugar en los primeros meses de 2010.

La concreción de este espectáculo, pensado exclusivamente para el directo y en el que la improvisación jugó un papel destacado sobre todo en los primeros momentos, cuenta como repertorio básico con las canciones de Leo y García a partir de poemas del propio Celso Emilio Ferreiro, cada uno de acuerdo con su estilo (en el caso de Leo con frecuencia a partir de versiones precedentes), pero estimulados y en diálogo con los estilos de sus compañeros de viaje. De todas formas, las canciones del grupo se han ido ampliando progresivamente en distintas direcciones. Por ejemplo en la conexión con repertorios consolidados de la resistencia latinoamericana (como la versión de "Hasta siempre", de Carlos Puebla) o con la actualización rítmica y letrística de "Cantiga pra unha antroidada", un alegato contra el autonomismo más sumiso que constituye un clásico del repertorio de Fuxan os Ventos.

Todas estas claves ayudan a clarificar la posición particular de Labregos do tempo dos sputniks en los campos poético y musical, determinada por la apuesta en espacios de producción y difusión alternativos a los propuestos desde el modelo normalizador, la 
importación de repertorios de resistencia política que funcionan en enclaves culturales considerados análogos y, finalmente, el aprovechamiento de la precariedad y de las grietas del campo cultural gallego para la actualización heterodoxa de determinada tradición literaria e ideológica.

\section{Fanny + Alexander}

Los proyectos musicales defendidos por el grupo de pop Fanny + Alexander (integrado por Efi Arias y Noel Feáns) representan, en buena medida, la voluntad articulada en determinados espacios de la cultura gallega por conseguir alcanzar la normalidad. El modelo de referencia se corresponde en el ámbito musical con la progresiva diversificación de estilos y propuestas, con el diálogo, con los discursos y modos de producción y difusión contemporáneos y, en definitiva, con la conformación de un público para los productos musicales creados en claves endógenas o autocentradas. El estilo del grupo encaja en esas coordenadas: pop con querencia por lo electrónico y abierto a la relación experimental con otros estilos. Con apertura, además, a determinadas fórmulas de creación y promoción como el vídeo-clip, infrecuentes en la música gallega.

Para entender las aspiraciones de Fanny + Alexander es imprescindible resaltar el papel desarrollado por Noel Feáns como planificador de la música emergente y alternativa en el panorama gallego. Se trata de un trabajo articulado en torno al netlabel www.aregueifa.net, creado en 2007 y que constituye la primera referencia de edición y difusión para aquellos grupos menos profesionalizados. Se asienta en unas bases que, además de la planificación innovadora, cuentan con el espíritu de la cultura libre (todos los discos del netlabel pueden ser descargados gratuitamente, incluidos los de Fanny + Alexander), con una independencia amplia en relación con las instituciones y con el afán de recuperar determinadas propuestas de la tradición gallega escasamente reconocidas.

En los tres discos que el grupo ha publicado (Lusco e fusco, 2006; Finais dos 70s, comezos dos 80s, 2007; Alfaias, 2009), la inmensa mayoría de las letras han sido producidas a partir de la adaptación de poemas contemporáneos, varios de ellos firmados por autores/as con poca o ninguna obra publicada ${ }^{15}$. Y aunque en algunos

\footnotetext{
${ }^{15}$ En el disco Finais dos 70s, comezos dos 80s (2007) contaron con poemas de María do Cebreiro, Lois Pereiro, Ricardo Carvalho Calero, Elvira Riveiro, Pilar Pallarés y Minus Bálido Rodríguez. El trabajo incluye también la pieza "Berlín", escrita e interpretada al modo del spoken word por María Lado y acompañada musicalmente por el grupo. En Alfaias (2009), Fanny + Alexander trabajó con textos de Mario Regueira, Emma Couceiro, Diego Ameixeiras, Lara Dopazo, Xiana Arias, Igor Lugrís, Pedro Lamas, Yolanda Castaño, Estíbaliz Espinosa, Daniel Salgado y Alberte Momán. Se apartarían de una posible agrupación generacional Lois Pereiro (poeta de acento punk e influencia centroeuropea, muerto en 1996), Ricardo Carvalho Calero (profesor, escritor e investigador con actividad ya antes de 1936 y afín a
} 
casos se ha optado por poemas escritos en la década de 1980, se trata de autores/as y obras que en ningún caso cuentan con un lugar central en el canon histórico de la poesía gallega. Las apuestas en este caso parecen ser otras: diversificar ese canon de acuerdo con criterios estilísticos, discursivos y temáticos renovados y, desde otro punto de vista, contribuir en una especie de movimiento alternativo, no tanto contra-cultural, articulado en torno a la ciudad de Compostela. Se trata, en todo caso, de selecciones muy heterogéneas y en las que parece primar una afinidad generacional que, obviamente, provoca el encuentro de poéticas no sólo diferentes sino claramente opuestas. Entre ellas, la poesía no-lírica y renovadoramente manifestataria de Daniel Salgado, la pulsión egótica y solo moderadamente provocadora de Yolanda Castaño, la poesía más convencionalmente política de Minus Bálido e Igor Lugris o, entre otras propuestas, el ludismo revoltoso y auto-referencial de Estíbaliz Espinosa.

En comparación con las propuestas de Rosalía 21 o de Labregos do tempo dos sputniks, el proyecto de Fanny + Alexander se posiciona de modo distinto frente a los ejes normalización/diferencia y centralidad/periferia. Su funcionalidad se orienta a la construcción de vías para la renovación y diversificación de un escenario musical que, en su carácter alternativo y en su deseo de homologarse a modelos internacionalizados, contribuya al proceso normalizador.

\section{Referencias bibliográficas}

ANGUEIRA, A., coord. (2009): Rosalía 21. Vigo, Xerais y Xunta de Galicia.

BOURDIEU, P. (1991): "Le champ littéraire", Actes de la Recherche en Sciences Sociales, 89 , pp. 3-46.

(1992): Les Règles de l'Art. Gènese et structure du champ littéraire. París, Seuil.

COCHÓN, I. (2001): "Unha proposta de superación da orde xeracional oitenta/noventa”, en D. VILLANUEVA, dir. y A. TARRÍO VARELA, coord., A literatura desde 1936 ata hoxe: Poesía e teatro. Enciclopedia Galicia, tomo XXXIII. A Coruña, Hércules de Ediciones, pp. 285-287.

EVEN-ZOHAR, I. (1990): Polysystem Studies. Volumen monográfico de Poetics Today, 11/1.

(2005): Papers in Culture Research. Tel Aviv, The Porter Chair of Semiotics y Tel Aviv University.

FIGUEROA, A. (2001): Nación, literatura e identidade. Vigo, Xerais.

los repertorios clásicos, muerto en 1990) y Pilar Pallarés (autora cuyo último libro, O livro das devoracións, fue publicado en 1994; en él culmina una particular renovación de los registros líricos y amorosos para la poesía escrita por mujeres). 
GONZÁLEZ-MILLÁN, X. (1994): Literatura e sociedade en Galicia (1975-1990). Vigo, Xerais.

- (2000): Resistencia cultural e diferencia histórica. Santiago de Compostela, Sotelo Blanco.

RODRÍGUEZ PRADO, M. F. (2004): “Inovaçons repertoriais no campo cultural galeguista na década de 70 e as transferências do mundo luso-afro-brasileiro", en A questão social no novo milénio. VIII Congresso Luso-Afro-Brasileiro de Ciências Sociais. Coimbra, 16, 17 e 18 de septiembre de 2004, en http://www.ces.uc.pt/lab2004/inscricao/pdfs/painel53/Felisa_Rodriguez_Prado.pdf [última consulta: 23-11-2011].

SAID, E. (1993): Culture and Imperialism. Nueva York, Knopf y Random House.

SOBRINO FREIRE, I. (2009): "Os prólogos a Cantares Gallegos e Follas Novas, manifestos por unha cultura da resistencia”, en Angueira (2009), pp. 37-42. 